\title{
COMPLETELY REGULAR MAPPINGS WITH LOCALLY COMPACT FIBER $\left({ }^{1}\right)$
}

\author{
BY \\ STEPHEN B. SEIDMAN
}

1. Introduction. Completely regular mappings were introduced by Dyer and Hamstrom in 1958 [2]. They are discrete analogues of locally trivial projections. We can thus ask for conditions under which a completely regular mapping is a fibration or is locally trivial. Some results along these lines were obtained in [2], and as a consequence of [8, p. 381], but in both cases the arguments apply only when the fiber is compact.

This paper will consider the case of locally compact fiber. We will prove that if $p: E \rightarrow B$ is completely regular with fiber $F$, where $F$ is locally compact and separable, and the homeomorphism group of $F$ (with a certain natural topology) is locally path-connected, then $p$ is a Serre fibration. If, in addition, $B$ is a finitedimensional $A N R$, then $p$ is locally trivial.

\section{Definitions and notation.}

(2.1) A continuous surjection $p: E \rightarrow B$ is completely regular if $E$ and $B$ are metric, and if for each $b \in B$ and $\varepsilon>0$, there exists $\delta(b, \varepsilon)>0$ such that if $d\left(b, b^{\prime}\right)<\delta$, there exists a homeomorphism $h: p^{-1}(b) \rightarrow p^{-1}\left(b^{\prime}\right)$ with $d(x, h(x))<\varepsilon$ for all $x \in p^{-1}(b)$.

A homeomorphism $g: X \rightarrow Y$ will always mean a homeomorphism onto $Y$.

The space $B$ will always be assumed to be connected. Thus all fibers are homeomorphic, and we will denote this common fiber by $F$.

(2.2) The homeomorphism group of $F$ will be denoted by $\mathscr{H}(F)$. If $F$ is compact, we give $\mathscr{H}(F)$ the compact-open topology. If $F$ is locally compact and separable, then the one-point compactification of $F, \hat{F}$, is metrizable. $\mathscr{H}(F)$ is naturally identified with $\mathscr{H}\left(\hat{F},{ }^{*}\right) \subseteq \mathscr{H}(\hat{F})$, the space of homeomorphisms of $\hat{F}$ fixing $\{*\}=$ $\hat{F}-F$. We will give $\mathscr{H}(F)$ the topology induced from $\mathscr{H}(\hat{F})$ by this identification. This is the g-topology of Arens [1].

(2.3) A continuous surjection $p: E \rightarrow B$ is generalized completely regular (g.c.r.) if, given $b \in B$ and an open cover $\left\{U_{\alpha}\right\}(\alpha \in A)$ of $p^{-1}(b)$, there exists an open neighborhood $V$ of $b$ such that if $b^{\prime} \in V$, then there exists a homeomorphism $h: p^{-1}(b) \rightarrow p^{-1}\left(b^{\prime}\right)$, such that for each $x \in p^{-1}(b),\{x, h(x)\} \subseteq U_{\alpha}$ for some $\alpha \in A$.

Received by the editors May 23, 1969.

(1) The results presented in this paper are contained in the author's doctoral dissertation, prepared at the University of Michigan under the direction of Professor Morton Brown. 
Observe that if $p: E \rightarrow B$ is g.c.r., while $E$ is separable metric and $B$ is metric, then $F$ is compact.

A topological space is locally $n$-connected $\left(L C^{n}\right)$ if, given $x \in X$ and an open neighborhood $U$ of $x$, then there exists an open set $V$ with $x \in V \subseteq U$, such that if $f: S^{m} \rightarrow V$ is a map $(m \leqq n)$, then $f$ extends to $F: B^{m+1} \rightarrow U$.

Let $\left\{S_{\alpha}\right\}(\alpha \in A)$ be a collection of subsets of $X .\left\{S_{\alpha}\right\}$ is equi-LC ${ }^{n}$ [7] if, given $x \in X$ and an open neighborhood $U$ of $x$, there exists an open set $V$ with $x \in V \subseteq U$, such that if $f: S^{m} \rightarrow V \cap S_{\alpha}$ is a map ( $m \leqq n$ and $\alpha \in A$ ), then $f$ extends to

$$
F: B^{m+1} \rightarrow U \cap S_{\alpha}
$$

Let $Y$ be a topological space, and let $\mathscr{P}(Y)$ denote the collection of subspaces of $Y$. A function $\varphi: X \rightarrow \mathscr{P}(Y)$ is called a lower semicontinuous carrier (l.s.c. carrier) [6] if, given $x \in X$ and an open subset $U$ of $Y$ with $\varphi(x) \cap U \neq \varnothing$, then there exists an open neighborhood $V$ of $x$, such that if $x^{\prime} \in V$, then $\varphi\left(x^{\prime}\right) \cap U \neq \varnothing$.

(2.4) Note that if $p: E \rightarrow B$ is continuous and open, then the function taking $b$ to $p^{-1}(b)$ is a l.s.c. carrier from $B$ to $\mathscr{P}(E)[6$, p. 382].

We quote a version of a result of Michael [7, Theorem 1.2, p. 563].

(2.5) Proposition. Suppose that $X$ is metric and that $Y$ is complete metric. Let $\varphi: X \rightarrow \mathscr{P}(Y)$ be a l.s.c. carrier, where $\varphi(x)$ is closed for each $x \in X$, and the collection $\{\varphi(x)\}(x \in X)$ is equi-LC ${ }^{n}$. Suppose also that $\operatorname{dim} Y \leqq n+1$. Finally let $A \subseteq X$ be closed, and let $f: A \rightarrow Y$ be continuous, with $f(a) \in \varphi(a)$ for all $a \in A$.

Then $f$ can be extended to a continuous $g: U \rightarrow Y$, where $U \supseteq A$ is open, and $g(x) \in \varphi(x)$ for all $x \in U$.

A map $p: E \rightarrow B$ is a Serre fibration if it has the PCHP in the sense of $\mathrm{Hu}[3$, p. 62]. $p$ is locally trivial if for some space $F$, each point $b \in B$ has an open neighborhood $U(b)$, such that there is a homeomorphism $\varphi_{U}: F \times U \rightarrow p^{-1}(U)$ with $p \varphi_{U}(x, c)=c$, for all $x \in F$ and $c \in U(b) . p$ is trivial if we can take $U=B . p: E_{1} \rightarrow B$ and $q: E_{2} \rightarrow B$ are equivalent if there exists a homeomorphism $h: E_{1} \rightarrow E_{2}$ such that $p=q h$.

If $X$ and $Y$ are metric spaces, then a metric on $X \times Y$ is given by $d\left((x, y),\left(x^{\prime}, y^{\prime}\right)\right)$ $=\max \left\{d\left(x, x^{\prime}\right), d\left(y, y^{\prime}\right)\right\}$. We will always use this metric for $X \times Y$, without further explicit mention.

3. Generalities on complete regularity. The following two propositions relate the concepts introduced in (2.1) and (2.3).

(3.1) Proposition. If $p: E \rightarrow B$ is g.c.r., and $E$ and $B$ are metric, then $p$ is completely regular.

Proof. Let $b \in B$ and $\varepsilon>0$ be given. Since $p^{-1}(b) \subseteq \bigcup_{x \in p^{-1}(b)} N_{\varepsilon / 2}(x)$, and $p$ is g.c.r., there exists an open neighborhood $V$ of $b$, as in (2.3). Let $\delta(b, \varepsilon)>0$ be such 
that $N_{\delta}(b) \subseteq V$. Then, if $d\left(b, b^{\prime}\right)<\delta$, we have $b^{\prime} \in V$, so that there exists a homeomorphism $h: p^{-1}(b) \rightarrow p^{-1}\left(b^{\prime}\right)$ such that, if $x \in p^{-1}(b),\{x, h(x)\} \subseteq N_{\varepsilon / 2}\left(x^{\prime}\right)$, for some $x^{\prime} \in p^{-1}(b)$. But then $d(x, h(x))<\varepsilon$. Q.E.D.

(3.2) Proposition. Suppose that $p: E \rightarrow B$ is completely regular with compact fiber. Then $p$ is g.c.r.

Proof. Let $\left\{U_{\alpha}\right\}(\alpha \in A)$ be an open cover of $p^{-1}(b)$. For each $x \in p^{-1}(b)$, find $\varepsilon(x)>0$ such that $N_{2 \varepsilon(x)}(x) \subseteq U_{\alpha}$, for some $\alpha \in A$. Since $\left\{N_{\varepsilon(x)}(x)\right\}\left(x \in p^{-1}(b)\right)$ is an open cover of the compact set $p^{-1}(b)$, we can find a finite subcover $\left\{N_{\varepsilon\left(x_{i}\right)}\left(x_{i}\right)\right\}$ $(i=1, \ldots, k)$. Put $\varepsilon=\min _{i}\left\{\varepsilon\left(x_{i}\right)\right\}$. Now let $V=N_{\delta(b, \varepsilon)}(b)$. If $b^{\prime} \in V$, there exists a homeomorphism $h: p^{-1}(b) \rightarrow p^{-1}\left(b^{\prime}\right)$, as in (2.1). But if $x \in p^{-1}(b)$, then $x \in N_{\varepsilon\left(x_{i}\right)}\left(x_{i}\right)$ for some $i$. Thus $d\left(x, x_{i}\right)<\varepsilon\left(x_{i}\right)$, so that $d\left(h(x), x_{i}\right)<\varepsilon+\varepsilon\left(x_{i}\right) \leqq 2 \varepsilon\left(x_{i}\right)$. Hence

$$
\{x, h(x)\} \subseteq U_{\alpha}
$$

for some $\alpha \in A$, and so $p$ is g.c.r., as claimed.

Thus, if the fiber is compact, complete regularity and generalized complete regularity are equivalent concepts. Since generalized complete regularity does not depend on the metrics used for $E$ and $B$, we see that complete regularity does not depend on the choice of metric, if the fiber is compact.

The next result will be important in the following section.

(3.3) Theorem. Let $p: E \rightarrow B$ be completely regular with fiber $F$, where $B$ and $F$ are locally compact. Then $E$ is locally compact.

We will first prove

(3.4) For each $x \in E$, we can find an open neighborhood $U$ of $x$, such that $\bar{U} \cap p^{-1}(b)$ is compact for each $b \in B$.

Proof. Let $x \in E$ be given. By hypothesis, $p^{-1}(p(x))$ is locally compact. Thus there must exist an open neighborhood $W$ of $x$ in $E$, such that

$$
\overline{W \cap p^{-1}(p(x))}
$$

is compact. We can find $\varepsilon>0$, such that

$$
\overline{N_{\varepsilon}(x)} \subseteq W .
$$

We now have that

$$
\overline{N_{\varepsilon}(x)} \cap p^{-1}(p(x)) \subseteq W \cap p^{-1}(p(x)) \subseteq \overline{W \cap p^{-1}(p(x))},
$$

so that

$$
\overline{N_{\varepsilon}(x)} \cap p^{-1}(p(x))
$$

is compact.

Let $\delta(p(x), \varepsilon / 2)$ be as in (2.1). Then define

$$
U=N_{\varepsilon / 2}(x) \cap p^{-1}\left(N_{\delta(p(x), \varepsilon / 2) / 2}(p(x))\right) .
$$


We claim that $U$ is the desired open set. Thus we must look at $\bar{U} \cap p^{-1}(b)$. Now observe that

$$
\bar{U} \subseteq \overline{N_{\varepsilon / 2}(x)} \cap p^{-1}\left(N_{\delta(p(x), \varepsilon / 2)}(p(x))\right) .
$$

Thus if $\bar{U} \cap p^{-1}(b) \neq \varnothing$, we must have $d(b, p(x))<\delta(p(x), \varepsilon / 2)$. Then we can find a homeomorphism $h: p^{-1}(b) \rightarrow p^{-1}(p(x))$, such that $d(z, h(z))<\varepsilon / 2$, for all $z \in p^{-1}(b)$. But if $z \in \bar{U} \cap p^{-1}(b)$, then $d(z, x) \leqq \varepsilon / 2$, and so $d(h(z), x)<\varepsilon$. Then we have

$$
h\left(\bar{U} \cap p^{-1}(b)\right) \subseteq N_{\varepsilon}(x) \cap p^{-1}(p(x)) .
$$

Since $h$ is a homeomorphism, $h\left(\bar{U} \cap p^{-1}(b)\right)$ is a closed subspace of a compact space, and is hence compact. Finally, $\bar{U} \cap p^{-1}(b)$ is compact, as asserted.

Proof of (3.3). Let $x \in E$ be given, and let $V$ be an open neighborhood of $x$ as in (3.4). Let $\varepsilon>0$ be such that $N_{\varepsilon}(x) \subseteq V$. Since $B$ is locally compact, we can find an open neighborhood $W$ of $p(x)$ such that $\bar{W}$ is compact.

Let $\mu>0$ be given. For each $c \in \bar{W}$, we have $\delta(c, \mu / 2)>0$. Let $\lambda(\mu)>0$ be a Lebesgue number for the cover $\left\{N_{\delta(c, \mu / 2)}(c)\right\}(c \in \bar{W})$ of $\bar{W}$. If $c, c^{\prime} \in \bar{W}$, and $d\left(c, c^{\prime}\right)<\lambda(\mu)$, then there exists a homeomorphism $h: p^{-1}(c) \rightarrow p^{-1}\left(c^{\prime}\right)$, such that $d(y, h(y))<\mu$ for all $y \in p^{-1}(c)$.

Put $\delta_{k}=\lambda(1 / k)$. We may assume that $\delta_{k} \leqq 1 / k$. Define

$$
U=N_{\varepsilon / 2}(x) \cap p^{-1}\left(N_{\delta_{1} / 4}(p(x))\right) \cap p^{-1}(W) .
$$

We claim that $\bar{U}$ is compact. It will suffice to show that each sequence in $\bar{U}$ has an accumulation point. We observe that

$$
\bar{U} \subseteq \overline{N_{\varepsilon / 2}(x)} \cap p^{-1}\left(N_{\delta_{1} / 2}(p(x))\right) \cap p^{-1}(\bar{W}) .
$$

Let $\left\{x_{i}\right\}(i=1,2, \ldots)$ be a sequence in $\bar{U}$. Then $\left\{p\left(x_{i}\right)\right\}$ is a sequence in $\bar{W}$, and so has an accumulation point in $\bar{W}$. By taking a subsequence, we may assume that $\left\{p\left(x_{i}\right)\right\}$ converges to $b \in \bar{W}$.

Define $n(i)=\max \left\{k \mid d\left(p\left(x_{i}\right), b\right)<\delta_{k}\right\}$. Since $d\left(p\left(x_{i}\right), b\right)<\delta_{1}$ for all $i, n(i)$ is always defined, and if $p\left(x_{i}\right) \neq b$, then $n(i)<\infty$. Thus there exists a homeomorphism $h^{i}: p^{-1}\left(p\left(x_{i}\right)\right) \rightarrow p^{-1}(b)$, such that $d\left(h^{i}(z), z\right)<1 / n(i)$ for all $z \in p^{-1}\left(p\left(x_{i}\right)\right)$. If $p\left(x_{i}\right)=b$, we take $h^{i}$ to be $i d_{p^{-1}(b)}$.

Let $N(\varepsilon)>2 / \varepsilon$ be an integer. Since $\left\{p\left(x_{i}\right)\right\}$ converges to $b$, there exists an integer $M(\varepsilon)$, such that $i \geqq M(\varepsilon)$ implies that $d\left(p\left(x_{i}\right), b\right)<\delta_{N(\varepsilon)}$. Thus if $i \geqq M(\varepsilon)$, we have that $n(i) \geqq N(\varepsilon)$, so that

$$
d\left(h^{i}\left(x_{i}\right), x_{i}\right)<1 / n(i) \leqq 1 / N(\varepsilon)<\varepsilon / 2 .
$$

But then, for $i \geqq M(\varepsilon)$, we now have $d\left(h^{i}\left(x_{i}\right), x\right)<\varepsilon / 2+d\left(x_{i}, x\right)<\varepsilon$. Thus we have

$$
\left\{h^{i}\left(x_{i}\right)\right\}_{i \geqq M(\varepsilon)} \subseteq N_{\varepsilon}(x) \cap p^{-1}(b) \subseteq \bar{V} \cap p^{-1}(b) .
$$


But $\bar{V} \cap p^{-1}(b)$ is compact, so that $\left\{h^{i}\left(x_{i}\right)\right\}$ has an accumulation point

$$
\bar{x} \in \bar{V} \cap p^{-1}(b) .
$$

We assert that $\left\{x_{i}\right\}$ also accumulates at $\bar{x}$. Suppose that $N>0$ is a given integer and $\eta>0$ is arbitrary. We have shown that for $i \geqq M(\eta), d\left(h^{i}\left(x_{i}\right), x_{i}\right)<\eta / 2$. Since $\left\{h^{i}\left(x_{i}\right)\right\}$ accumulates at $\bar{x}$, we can find an integer $n>\max \{N, M(\eta)\}$, with

$$
d\left(h^{n}\left(x_{n}\right), \bar{x}\right)<\eta / 2 .
$$

But then $d\left(x_{n}, \bar{x}\right)<\eta$. Thus $\left\{x_{i}\right\}$ accumulates at $\bar{x}$. Since every sequence in $\bar{U}$ has an accumulation point, $\bar{U}$ is countably compact and hence compact. Thus $E$ is locally compact, as was to be shown.

Suppose now that $p: E \rightarrow B$ is completely regular, with fiber $F$, and that $f: A \rightarrow B$ is continuous, where $A$ is a metric space. Then we can define the pullback

$$
f^{*}(E)=\{(a, e) \mid f(a)=p(e)\} \subseteq A \times E .
$$

$f^{*}(E)$ has a natural metric, and we will define $f^{*}(p): f^{*}(E) \rightarrow A$ by $\left(f^{*}(p)\right)(a, e)=a$.

(3.5) Proposition. $f^{*}(p)$ is completely regular, with fiber $F$.

Proof. Let $a \in A$ and $\varepsilon>0$ be given. Then $\delta(f(a), \varepsilon)>0$. $f$ is continuous, so that $f^{-1}\left(N_{\delta(f(a), \varepsilon)}(f(a))\right)$ is an open neighborhood of $a$. Therefore we can find $\delta^{\prime}(a, \varepsilon)>0$, such that

$$
N_{\delta^{\prime}(a, \varepsilon)}(a) \subseteq f^{-1}\left(N_{\delta(f(a), \varepsilon)}(f(a))\right) .
$$

We can certainly require that $\delta^{\prime}(a, \varepsilon)<\varepsilon$.

Now let $a^{\prime} \in A$, with $d\left(a, a^{\prime}\right)<\delta^{\prime}(a, \varepsilon)$. Then $d\left(f(a), f\left(a^{\prime}\right)\right)<\delta(f(a), \varepsilon)$, so that there exists a homeomorphism $h: p^{-1}(f(a)) \rightarrow p^{-1}\left(f\left(a^{\prime}\right)\right)$ with $d(x, h(x))<\varepsilon$ for all $x \in p^{-1}(f(a))$. Define $h^{\prime}:\left(f^{*}(p)\right)^{-1}(a) \rightarrow\left(f^{*}(p)\right)^{-1}\left(a^{\prime}\right)$ by $h^{\prime}(a, e)=\left(a^{\prime}, h(e)\right)$. Now $d\left((a, e),\left(a^{\prime}, h(e)\right)\right)<\varepsilon$, for all $(a, e) \in\left(f^{*}(p)\right)^{-1}(a)$. Since $h^{\prime}$ is a homeomorphism, it follows that $f^{*}(p)$ is completely regular, with fiber $F$.

We conclude this section with the simple observation

(3.6) If $p: E \rightarrow B$ is completely regular, then $p$ is an open map.

4. The case of locally compact fiber. We have observed that if the fiber is compact, complete regularity does not depend on the metrics used. However, we will now give a complete metric for $R^{2}$, equivalent to the usual Euclidean metric, with respect to which $\mathrm{pr}_{1}: R^{2} \rightarrow R^{1}$ is not completely regular.

Let $d\left(\left(t_{1}, u_{1}\right),\left(t_{2}, u_{2}\right)\right)=\left|t_{1}-t_{2}\right|+\left|u_{1}-u_{2}\right|$. Define a homeomorphism $h: R^{2} \rightarrow R^{2}$ by $h(t, u)=\left(t\left(1+u^{2}\right)^{1 / 2}, u\right)$. Then let $\bar{d}\left(\left(t_{1}, u_{1}\right),\left(t_{2}, u_{2}\right)\right)=d\left(h\left(t_{1}, u_{1}\right), h\left(t_{2}, u_{2}\right)\right) . \bar{d}$ is evidently a complete metric for $R^{2}$ that is equivalent to the Euclidean metric. However, since $h$ takes vertical lines into hyperbolas, it is clear that $\operatorname{pr}_{1}$ is not completely regular with respect to $\bar{d}$.

Hence the noncompact case is intrinsically more difficult than the compact case. Suppose now that $p: E \rightarrow B$ is completely regular, with respect to some metric 
on $E$. Define a new space

$$
\hat{E}=E \cup \bigcup_{b \in B}\left\{\hat{e}_{b}\right\}
$$

and a function $\hat{p}: \hat{E} \rightarrow B$ by $\hat{p} \mid E=p$, and $\hat{p}\left(\hat{e}_{b}\right)=b$. Give $\hat{E}$ the topology defined by the basis consisting of the open sets of $E$ and the sets of the form $(\hat{E}-K) \cap \hat{p}^{-1}(U)$, where $K \subseteq E$ is compact and $U \subseteq B$ is open.

It easily follows that $\hat{p}$ is continuous, and that the topology induced from $\hat{E}$ on $\hat{p}^{-1}(b)$ is that of the one-point compactification of $p^{-1}(b)$. A similar, but more complicated, construction is used by Kim [5], in dealing with Hurewicz fibrations. We will first prove

(4.1) Proposition. $\hat{p}: \hat{E} \rightarrow B$ is g.c.r., and moreover the homeomorphisms $h: \hat{p}^{-1}(b) \rightarrow \hat{p}^{-1}\left(b^{\prime}\right)$ required by (2.3) can be chosen so that $h\left(\hat{e}_{b}\right)=\hat{e}_{b^{\prime}}$.

Proof. Let $\left\{W_{\alpha}\right\}(\alpha \in A)$ be an open cover of $\hat{p}^{-1}(b)$. Since $\hat{p}^{-1}(b)$ is compact, we can find a refinement of this cover of the form

$$
\begin{aligned}
W_{1}^{\prime} & =\left(\hat{p}^{-1}\left(U_{1}\right)\right) \cap\left(\hat{E}-K_{1}\right), \ldots, W_{k}^{\prime}=\left(\hat{p}^{-1}\left(U_{k}\right)\right) \cap\left(\hat{E}-K_{k}\right), \\
W_{k+1}^{\prime} & =V_{k+1}, \ldots, W_{n}^{\prime}=V_{n},
\end{aligned}
$$

where $K_{i} \subseteq E$ is compact, $U_{i} \subseteq B$ is open and $V_{j} \subseteq E$ is open $(i=1, \ldots, k ; j=$ $k+1, \ldots, n)$. It will evidently suffice to verify the condition of (2.3) for this refinement.

Write $V_{i}=E-K_{i}$ for $2 \leqq i \leqq k$, so that $p^{-1}(b)$ is covered by $E-K_{1}, V_{2}, \ldots, V_{n}$, where $V_{i}(2 \leqq i \leqq n)$ is open and $K_{1}$ is compact. Evidently $p^{-1}(b) \cap K_{1} \subseteq \bigcup_{i=2}^{n} V_{i}$. For each $x \in p^{-1}(b) \cap K_{1}$, we can find $\varepsilon(x)>0$, such that $N_{\varepsilon(x)}(x) \subseteq V_{i}$, for some $i$. Clearly there exists a finite cover $\left\{N_{\varepsilon\left(x_{i}\right) / 2}\left(x_{i}\right)\right\}(i=1, \ldots, m)$ of $p^{-1}(b) \cap K_{1}$. Let $\varepsilon=\min \left\{\varepsilon\left(x_{i}\right)\right\}$. Certainly $\varepsilon>0$.

Suppose now that $h: p^{-1}(b) \rightarrow p^{-1}\left(b^{\prime}\right)$ is a homeomorphism such that $d(x, h(x))$ $<\varepsilon / 2$ for all $x \in p^{-1}(b)$. Then $d(x, h(x))<\varepsilon\left(x_{i}\right) / 2$ for $i=1, \ldots, m$. Now

$$
p^{-1}(b) \cap K_{1} \subseteq \bigcup_{i=1}^{m} N_{\varepsilon\left(x_{i}\right) / 2}\left(x_{i}\right),
$$

and so if $x \in p^{-1}(b) \cap K_{1}$, then $d\left(x, x_{i}\right)<\varepsilon\left(x_{i}\right) / 2$ for some $i$. Thus $d\left(h(x), x_{i}\right)<$ $\varepsilon\left(x_{i}\right) / 2+\varepsilon\left(x_{i}\right) / 2=\varepsilon\left(x_{i}\right)$. Thus $\{x, h(x)\} \subseteq N_{\varepsilon\left(x_{i}\right)}\left(x_{i}\right) \subseteq V_{j}$, for some $j(2 \leqq j \leqq n)$.

Since $K_{1}$ is compact, we can choose $\eta>0$ such that

$$
\eta<d\left(K_{1}, p^{-1}(b)-\bigcup_{i=1}^{m} N_{\varepsilon\left(x_{i}\right) / 2}\left(x_{i}\right)\right)
$$

Define $\mu=\delta(b, \min (\eta, \varepsilon / 2))$, and let $U=N_{\mu}(b)$. If $b^{\prime} \in U$, there exists a homeomorphism $h: p^{-1}(b) \rightarrow p^{-1}\left(b^{\prime}\right)$ with $d(h(x), x)<\min (\eta, \varepsilon / 2)$ for all $x \in p^{-1}(b)$. Thus if $x \in \bigcup_{i=1}^{m} N_{\varepsilon\left(x_{i}\right) / 2}\left(x_{i}\right)$, it follows that $\{x, h(x)\} \subseteq V_{j}$, for some $j$.

But if $x \notin \bigcup_{i=1}^{m} N_{\varepsilon\left(x_{i}\right) / 2}\left(x_{i}\right)$, then clearly $h(x) \notin K_{1}$, since $d(h(x), x)<\eta$. Hence $\{x, h(x)\} \subseteq E-K_{1}$. 
Finally, put $V=U \cap\left(\bigcap_{t=1}^{k} U_{t}\right) . V$ is an open neighborhood of $b$. We claim that $V$ is the open set required by (2.3). Thus let $b^{\prime} \in V$. Since $b^{\prime} \in U$, we can find a homeomorphism $h: p^{-1}(b) \rightarrow p^{-1}\left(b^{\prime}\right)$ as above. Define $\hat{h}: \hat{p}^{-1}(b) \rightarrow \hat{p}^{-1}\left(b^{\prime}\right)$ by $\hat{h}(x)=h(x)$ for $x \in p^{-1}(b)$, and $\hat{h}\left(\hat{e}_{b}\right)=\hat{e}_{b^{\prime}}$. Now if $x \in p^{-1}(b)$, then $\{x, \hat{h}(x)\} \subseteq \hat{E}-K_{1}$ or $\{x, \hat{h}(x)\} \subseteq V_{j}$ for some $j$, as shown above. Since $\left\{b, b^{\prime}\right\} \subseteq \bigcap_{t=1}^{k} U_{t}$, we see that if $x \in p^{-1}(b)$, then $\{x, \hat{h}(x)\} \subseteq W_{i}^{\prime}$ for some $i$. But $\left\{\hat{e}_{b}, \hat{e}_{b^{\prime}}\right\} \subseteq\left(\hat{E}-K_{1}\right) \cap \hat{p}^{-1}\left(U_{1}\right)$, so that $\hat{h}$ satisfies the condition of (2.3).

It remains to show that $\hat{h}$ is a homeomorphism. Basic open sets in $\hat{p}^{-1}(b)$ are of the form $U \cap \hat{p}^{-1}(b)$ and $(\hat{E}-K) \cap \hat{p}^{-1}(b)$, where $U \subseteq E$ is open and $K \subseteq E$ is compact. Now $\hat{h}\left(U \cap \hat{p}^{-1}(b)\right)=h\left(U \cap p^{-1}(b)\right)$, which is open in $p^{-1}\left(b^{\prime}\right)$ (and hence in $\left.\hat{p}^{-1}\left(b^{\prime}\right)\right)$ since $h$ is a homeomorphism. Also,

$$
\hat{h}\left((\hat{E}-K) \cap \hat{p}^{-1}(b)\right)=h\left((E-K) \cap p^{-1}(b)\right) \cup\left\{\hat{e}_{b^{\prime}}\right\} .
$$

But $h\left((E-K) \cap p^{-1}(b)\right)=\left(E-h\left(K \cap p^{-1}(b)\right)\right) \cap p^{-1}\left(b^{\prime}\right)$, and $h\left(K \cap p^{-1}(b)\right)$ is compact. Thus we see that

$$
\hat{h}\left((\hat{E}-K) \cap \hat{p}^{-1}(b)\right)=\left(\hat{E}-h\left(K \cap p^{-1}(b)\right)\right) \cap \hat{p}^{-1}\left(b^{\prime}\right),
$$

which is open in $\hat{p}^{-1}\left(b^{\prime}\right)$. Thus $\hat{h}$ is an open map. Similarly, we see that $\hat{h}$ is continuous, and so $\hat{h}$ is a homeomorphism. Q.E.D.

We will now consider the topology of $\hat{E}$. We have

(4.2) Proposition. If $F$ and $B$ are locally compact and separable, then $\hat{E}$ is metrizable.

Proof. It follows easily from (3.3) and (3.6) that $E$ is locally compact and separable. To see that $E$ is Hausdorff, observe first that any two points of $E$ can be separated by open sets in $\hat{E}$. Since $b$ and $b^{\prime}$ can be separated in $B, \hat{e}_{b}$ and $\hat{e}_{b^{\prime}}$ can be separated in $\hat{E}$. If $x \in E$, we can find an open set $W$ in $E$, with $(\bar{W})_{E}$ compact, where $(\bar{W})_{E}$ denotes the closure of $W$ in $E$. Then, if $b \in B, \hat{e}_{b} \in \hat{E}-(\bar{W})_{E}$, which is open in $\hat{E}$, so that $x$ can be separated from $\hat{e}_{b}$.

We will next show that $\hat{E}$ is regular. If $A \subseteq E$ is such that $(\bar{A})_{E}$ is compact, then $(\bar{A})_{E}=(\bar{A})_{E}$. Thus if $x \in U$, where $U$ is open in $E$, we can find $V$, open in $E$, with $x \in V \subseteq(\bar{V})_{E} \subseteq U$. It only remains to consider the case $\hat{e}_{b} \in(\hat{E}-K) \cap \hat{p}^{-1}(U)$, for $K$ compact and $U$ open. We can find an open set $W$ in $B$, with $b \in W \subseteq \bar{W} \subseteq U$. Since $E$ is locally compact, we can find a compact set $K^{\prime}$, with $K \subseteq$ int $\left(K^{\prime}\right) \subseteq K^{\prime}$. We have

$$
\hat{e}_{b} \in\left(\hat{E}-K^{\prime}\right) \cap \hat{p}^{-1}(W) \subseteq(\hat{E}-K) \cap \hat{p}^{-1}(U) .
$$

But

$$
\begin{aligned}
\overline{\left(\left(\hat{E}-K^{\prime}\right) \cap \hat{p}^{-1}(W)\right)} & \subseteq \overline{\left(\hat{E}-K^{\prime}\right)} \cap \overline{\hat{p}^{-1}(W)} \subseteq\left(\hat{E}-\operatorname{int}\left(K^{\prime}\right)\right) \cap \hat{p}^{-1}(\bar{W}) \\
& \subseteq(\hat{E}-K) \cap \hat{p}^{-1}(U) .
\end{aligned}
$$

Thus $\hat{E}$ is regular, as claimed. 
Finally, we will show that $\hat{E}$ is second countable. $E$ and $B$ are separable metric spaces, and hence are second countable. Let $\left\{V_{i}\right\}(i=1,2, \ldots)$ be a basis for $B$, and let $\left\{U_{i}\right\}(i=1,2, \ldots)$ be a basis for $E$ such that each $\bar{U}_{i}$ is compact. Then we claim that the collection $\left\{(\hat{E}-M) \cap \hat{p}^{-1}\left(V_{j}\right)\right\}$, where $M$ runs through all finite unions of the sets $\left\{U_{i}\right\}$, together with the collection $\left\{U_{i}\right\}$, is a basis for $\hat{E}$.

To see this we need only look at $\hat{e}_{b} \in(\hat{E}-K) \cap \hat{p}^{-1}(N)$, where $K$ is compact and $N$ is open. Since $K$ is compact, we can cover $K$ by $U_{i_{1}}, \ldots, U_{i_{n}}$; then

$$
K \subseteq \bigcup_{t=1}^{n} \bar{U}_{i_{t}}
$$

There exists $V_{i}$ with $b \in V_{i} \subseteq N$. Thus

$$
\hat{e}_{b} \in\left(\hat{E}-\bigcup_{t=1}^{n} \bar{U}_{i_{t}}\right) \cap \hat{p}^{-1}\left(V_{i}\right) \subseteq(\hat{E}-K) \cap \hat{p}^{-1}(N),
$$

and therefore we have found a countable basis for $E$.

Since $\hat{E}$ is regular and second countable, we see by Urysohn's metrization theorem that $\hat{E}$ is metrizable.

We now have the corollary

(4.3) $\hat{p}: \hat{E} \rightarrow B$ is completely regular, and moreover the homeomorphism

$$
\hat{h}: \hat{p}^{-1}(b) \rightarrow \hat{p}^{-1}\left(b^{\prime}\right)
$$

required by (2.1) can be chosen so that $\hat{h}\left(\hat{e}_{b}\right)=\hat{e}_{b^{\prime}}$.

Proof. This follows from (3.1), (4.1), and (4.2).

Suppose now that, without any assumptions on $p$, we have that $\hat{p}: \hat{E} \rightarrow B$ is g.c.r., while $\hat{E}$ is metrizable. Then $\hat{p}$ is completely regular, and the metric on $\hat{E}$ induces a metric on $E$, with respect to which $p$ is completely regular. Thus we have

(4.4) Proposition. Suppose that $p: E \rightarrow B$ is a continuous surjection, where $E$ is locally compact separable metric, and $B$ is metric. Then $p$ is completely regular with respect to some (equivalent) metric on $E$ if and only if $\hat{p}: \hat{E} \rightarrow B$ is g.c.r.

Proof. We have proved "only if" in (4.1). To prove "if", it will suffice to show that $\hat{E}$ is metrizable. But this follows as in (4.2), since $E$ is locally compact and separable.

Therefore complete regularity for $p$ (with respect to some metric) is equivalent to generalized complete regularity for $\hat{p}$, if $E$ is locally compact and separable. Thus we need only consider the situation where the fiber is compact.

5. Proof of the main theorems. We will now show that a completely regular mapping with locally compact fiber, under the proper circumstances, is a fibration or is locally trivial. First we have

(5.1) Proposition. Let $p: E \rightarrow I$ be completely regular, with fiber $F$, where $F$ is locally compact and separable, and $\mathscr{H}(F)$ is $L C^{0}$. Then $p$ is trivial. 
Proof. As in the preceding section, form the metric space $\hat{E}$. By (3.3), $\hat{E}$ is locally compact, so that $\hat{E}$ has a complete metric. Since $\hat{F}$ is compact, we see that $p: \hat{E} \rightarrow I$ is completely regular with respect to this complete metric. $\hat{p}$ has fiber $\hat{F}$.

From (2.2), we see that $\mathscr{H}\left(\hat{F},{ }^{*}\right)$ is $L C^{0}$. Let $\mathscr{H}_{t}$ be the space of homeomorphisms of $\left(\hat{F},{ }^{*}\right)$ onto $\left(\hat{p}^{-1}(t), \hat{e}_{t}\right)$. Put $\mathscr{H}=\bigcup_{t \in I} \mathscr{H}_{t} . \mathscr{H} \subseteq \hat{E}^{\hat{F}}$, and we give $\mathscr{H}$ the topology induced from the compact-open topology on $\hat{E}^{\hat{F}} \cdot \bar{p}: \mathscr{H} \rightarrow I$ is defined by $\bar{p}\left(\mathscr{H}_{t}\right)=t$.

It easily follows that $\bar{p}$ is completely regular, and hence that $\bar{p}$ is an open map. Thus, by (2.4), the function that takes $t$ to $\mathscr{H}_{t}$ is a lower semicontinuous carrier from $I$ to $\mathscr{P}\left(\hat{E}^{f}\right)$. By making minor changes in [2, Lemma 2], we can show that $\mathscr{H}$ is topologically complete. But since $\mathscr{H}\left(\hat{F},{ }^{*}\right)$ is $L C^{0}$ and $\bar{p}$ is completely regular, we see [2, Lemma 3] that $\left\{\mathscr{H}_{t}\right\}(t \in I)$ is equi- $L C^{0}$.

We can now apply (2.5) to get, for each $t \in I$, an open neighborhood $U(t)$ of $t$, and a continuous function $s_{t}: U(t) \rightarrow \mathscr{H}$, such that $s_{t}\left(t^{\prime}\right) \in \mathscr{H}_{t^{\prime}}$ for all $t^{\prime} \in U(t)$.

Now define a map $\bar{s}_{t}: \hat{F} \times U(t) \rightarrow \hat{p}^{-1}(U(t))$ by

$$
\bar{s}_{t}\left(x, t^{\prime}\right)=\left(s_{t}\left(t^{\prime}\right)\right)(x), \text { for all } t^{\prime} \in U(t) \text { and } x \in F .
$$

This map satisfies $\hat{p}\left(\bar{s}_{t}\left(x, t^{\prime}\right)\right)=t^{\prime}$ for all $t^{\prime} \in U(t)$ and $x \in \hat{F}$. We also have $\bar{s}_{t}\left({ }^{*}, t^{\prime}\right)$ $=\hat{e}_{t^{\prime}}$ for all $t^{\prime} \in U(t)$. It is clear that $\bar{s}_{t}$ is a homeomorphism. Thus we have

$$
\bar{s}_{t} \mid F \times U(t): F \times U(t) \rightarrow p^{-1}(U(t)),
$$

which is also a homeomorphism. Thus $p: E \rightarrow I$ is locally trivial. But since $I$ is contractible, it follows that $[9$, p. 53$] p$ is trivial, as was to be shown.

We next have

(5.2) Proposition. Let $p: E \rightarrow I^{n}$ be completely regular, with fiber $F$, where $F$ is locally compact and separable, and $\mathscr{H}(F)$ is $L C^{0}$. Then $p$ is trivial.

Proof. As in the preceding proposition, we look at $\hat{p}: \hat{E} \rightarrow I^{n}$. We then follow the argument in [2, Theorem 5], always keeping track of the point added to each fiber in the construction of $\hat{E}$. The result will follow as in the proof of (5.1).

We can draw the immediate corollary

(5.3) Let $p: E \rightarrow M^{n}$ be completely regular, with fiber $F$, where $F$ is locally compact and separable, $\mathscr{H}(F)$ is $L C^{0}$, and $M^{n}$ is an n-manifold. Then $p$ is locally trivial.

So far, we have generalized the results of [2] to the case of locally compact fiber. The final step is to apply the pullback construction. We first prove

(5.4) THEOREM. Suppose that $p: E \rightarrow B$ is completely regular with fiber $F$, where $F$ is locally compact and separable, $\mathscr{H}(F)$ is $L C^{0}$, and $B$ is a finite-dimensional $A N R$. Then $p$ is locally trivial.

Proof. Since $\operatorname{dim} B<\infty$, we can embed $B$ in some Euclidean space $R^{k}[4$, p. 60]. But $B$ is an $A N R$, so that we can find a retraction $r: M^{k} \rightarrow B$, where $M^{k}$ is an 
open neighborhood of $B$ in $R^{k}$. Let $i: B \subseteq M^{k}$; thus we have $r i(b)=b$ for all $b \in B$. Now, by (5.3), $r^{*}(p): r^{*}(E) \rightarrow M^{k}$ is completely regular, with fiber $F$. Thus $r^{*}(p)$ is locally trivial. But then $i^{*}\left(r^{*}(p)\right)$ is locally trivial. Since $i^{*}\left(r^{*}(p)\right)$ is equivalent to $(r i)^{*}(p)$, we see that $i^{*}\left(r^{*}(p)\right)$ is equivalent to $1_{B}^{*}(p)$, which is in turn equivalent to $p$. Hence $p$ is locally trivial, as claimed.

Thus we have proved one of the major results claimed in the introduction. To prove the other, we will use a similar method.

(5.5) TheOREM. Let $p: E \rightarrow B$ be completely regular, with fiber $F$, where $F$ is locally compact and separable, and $\mathscr{H}(F)$ is $L C^{0}$. Then $p$ is a Serre fibration.

Proof. Since we must lift homotopies of cells, it will suffice to consider a diagram of the following sort:

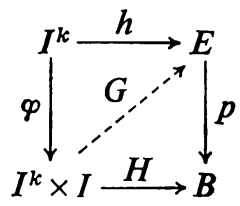

where $(x, 0)=\varphi(x)$, and $p h(x)=H \varphi(x)$ for all $x \in I^{k}$. We must construct a map $G: I^{k} \times I \rightarrow E$, such that $p G(x, t)=H(x, t)$ and $G(x, 0)=h(x)$, for all $x \in I^{k}$ and $t \in I$. Consider the map $H^{*}(p): H^{*}(E) \rightarrow I^{k} \times I$. This map is completely regular, with fiber $F$; hence it is trivial by (5.2). Now a trivial map is certainly a Serre fibration. Define $\bar{h}: I^{k} \rightarrow H^{*}(E)$ by $\bar{h}(x)=(x, 0, h(x))$. Since $H^{*}(p)(\bar{h}(x))=\varphi(x)$, we have the commutative diagram

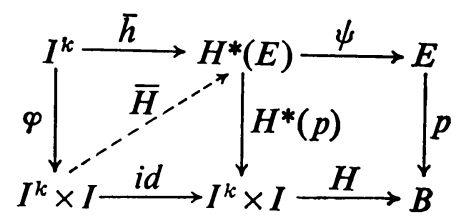

where $\psi$ is defined by $\psi(x, t, e)=e$. But then we can find $\bar{H}: I^{k} \times I \rightarrow H^{*}(E)$, such that $\left(H^{*}(p) \circ \bar{H}\right)(x, t)=(x, t)$, and $\bar{H}(x, 0)=\bar{h}(x)$, for all $x \in I^{k}$ and $t \in I$. Define $G: I^{k} \times I \rightarrow E$ by $G=\psi \circ \bar{H}$. It is easily verified that $G$ has the desired properties.

\section{REFERENCES}

1. R. Arens, Topologies for homeomorphism groups, Amer. J. Math. 68 (1946), 593-610. MR 8, 479.

2. E. Dyer and M.-E. Hamstrom, Completely regular mappings, Fund. Math. 45 (1958), 103-118. MR 19, 1187.

3. S. T. Hu, Homotopy theory, Pure and Appl. Math. vol. 8, Academic Press, New York, 1959. MR 21 \#5186. 
4. W. Hurewicz and H. Wallman, Dimension theory, Princeton Univ. Press, Princeton, N. J., 1941. MR 3, 312.

5. S. K. Kim, Local triviality of Hurewicz fiber maps, Trans. Amer. Math. Soc. 135 (1969), 51-67. MR 38 \#1679.

6. E. Michael, Continuous selections. I, Ann. of Math. (2) 63 (1956), 361-382. MR 17, 990.

7. - Continuous selections. II, Ann. of Math. (2) 64 (1956), 562-580. MR 18, 325.

8. - Continuous selections. III, Ann. of Math. (2) 65 (1957), 375-390. MR 18, 750.

9. N. Steenrod, The topology of fibre bundles, Princeton Univ. Press, Princeton, N. J., 1951. MR 12, 522.

NeW YoRK UNIVERSITY,

New York, New York 